\title{
FSI Modeling of a Propulsion System Based on Compliant Hydrofoils in a Tandem Configuration
}

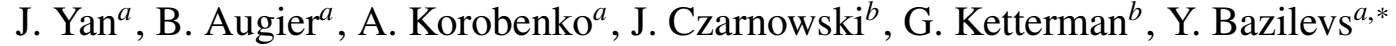 \\ ${ }^{a}$ Department of Structural Engineering, University of California, San Diego, La Jolla, CA 92093, USA \\ ${ }^{b}$ Hobie Cat Company, Oceanside, CA 92056, USA
}

\begin{abstract}
Fluid-structure interaction (FSI) analysis of the Mirage Drive, a bioinspired propulsion system utilizing compliant hydrofoils designed and built by Hobie Cat $\mathrm{Co}$, is performed. The current work continues a recent experimental and numerical FSI study of a single compliant hydrofoil, and presents, for the first time, full-scale FSI simulation of two compliant hydrofoils in a tandem configuration. The underlying computational challenges are addressed by means of core FSI methods (i.e., the ALE-VMS formulation, weakly enforced no-slip boundary conditions, the sliding-interface technique, and a quasi-direct coupling strategy), as well as special FSI techniques developed for this problem class. Details of the computational framework are discussed, and the computational results are validated using data from field tests, which are also described in the manuscript.
\end{abstract}

Keywords: Hydrofoil propulsion, Fluid-structure interaction, Bioinspired engineering, ALE-VMS, IGA

\section{Introduction}

Nowadays, the concept of bioinspired (or biomimetic) engineering is widely used in the design of propulsive and energy harvesting systems. Examples of such systems include micro aerial vehicles (MAVs) [61, 63, 67, 77, 82], vertical-axis wind turbines (VAWTs) placed in arrays [25, 45], and recreational water sports vehicles, like kayaks [6]. The main hypothesis behind the bioinspired engineering concept is that efficiency of engineering systems or devices

${ }^{*}$ Corresponding author; e-mail: yuri@ ucsd.edu; tel: +1-858-534-3663

Preprint submitted to Elsevier

July 5, 2015 
may be improved by mimicking the shape, structure, and motion of various species (e.g., birds, insects, or fish), which have these characteristics optimized in the long span of their evolution. In an effort to fully understand the underlying mechanisms leading to optimized performance, in recent years there has been a significant increase in the research focusing on aerodynamic, hydrodynamic, and structural modeling, simulation, and testing of bioinspired systems and devices $[26,47,52,57,58,61,63,65,66,77]$.

In the present paper, we perform fluid-structure interaction (FSI) analysis of a bioinspired propulsion system utilizing compliant hydrofoils. This work, which is an extension of a recent experimental and numerical FSI study of a single compliant hydrofoil [6], presents, for the first time, full-scale FSI simulation of two compliant hydrofoils in a tandem configuration. We note that experimental and numerical studies of hydrofoils were reported previously in the literature (see, e.g., [23, 44, 46, 54]). However, simulations reported in these references were performed with significant simplifications in the foil geometry modeled, mechanics represented, and operating conditions employed.

In order to carry out the hydrofoil FSI simulations, several computational challenges need to be addressed: 1 . The flow Reynolds number based on the cord length and relative flow speed is about 0.5 million, resulting in thin, turbulent boundary layers near compliant foil surfaces requiring accurate approximation; 2 . The tandem hydrofoil configuration includes an upstream (or front) and downstream (or back) foils that interact with one another. Furthermore, the foils move in opposite directions and periodically come in close proximity to one another. (The foils considered in the present paper are $37 \mathrm{~cm}$ in length and the closest clearance between them is less than $2.6 \mathrm{~cm}$.) This situation requires advanced mesh handling strategies; 3 . The foils are highly flexible and undergo large deformation in addition to rigid rotation. Capturing the details of that deformation, i.e., the foil twisting motions, is very important for accurate prediction of hydrodynamic loads and assessment of the propulsion system efficiency; 4. Since the foils are relatively light and operate in a hydrodynamic environment, the added mass effect is significant and precludes the use of simple-to-implement block-iterative FSI solution strategies [88-90, 92].

The above outlined challenges are akin to those involved in other FSI applications [19, 20], 
such as the spacecraft aerodynamics [69], FSI modeling of spacecraft parachutes [68, 71, 7376, 80, 81, 84], sails [4, 5, 91], cardiovascular fluid mechanics and FSI [10, 13, 14, 30, 49, 60, $61,64,70,72,78,79,83]$, pulsatile ventricular assist devices [48, 50, 51], and bioprosthetic heart valves $[32,33,38]$, and are addressed in the present paper in order to carry out the tandem hydrofoil FSI simulations. A moving-mesh FSI methodology is employed in the present work. The hydrodynamics is simulated using a finite-element-based ALE-VMS technique [12, $62]$ in combination with weakly enforced essential boundary conditions $[15,17,18,28]$ and boundary-layer meshing. These address the challenges associated with turbulence modeling, good resolution of boundary-layer phenomena, and accurate prediction of the hydrodynamic loads on the foils. The relative motion of the foils is handled through a sliding-interface technique $[16,31]$. The foil structural modeling and discretization makes use of isogeometric analysis (IGA) [24, 34]. A recently proposed rotation-free shell [41, 42] coupled with a beam/cable formulation [53] is employed, resulting in a smooth deformation of the foil surface during the simulation. The FSI formulation is based on the augmented Lagrangian technique with formal elimination of the Lagrange multiplier variable, and enables coupling of FEM and IGA with a nonmatching fluid-structure interface [11]. Finally, the high added mass in the problem is handled by means of a quasi-direct coupling strategy [88-90, 92] and its special implementation based on a matrix-free approach [20].

The paper is outlined as follows. In section 2, we describe the field test setup for the tandem hydrofoil configuration and report the measurements performed. In section 3, we review the governing equations of the FSI problem in the strong form and discuss the discretization techniques and solution strategies employed. In section 4, we describe the computational setup and present the results of the FSI simulation including comparison with field-test data and detailed discussion of the phenomena observed. In section 5, we draw conclusions.

\section{Field Test Setup and Measurements}

In this section, we briefly describe the propulsion system analyzed, which is the Mirage Drive designed and built by Hobie Cat Co. For more details the reader is referred to [6], where such a measurement system is presented in detail. Here the field test procedures are adapted for 


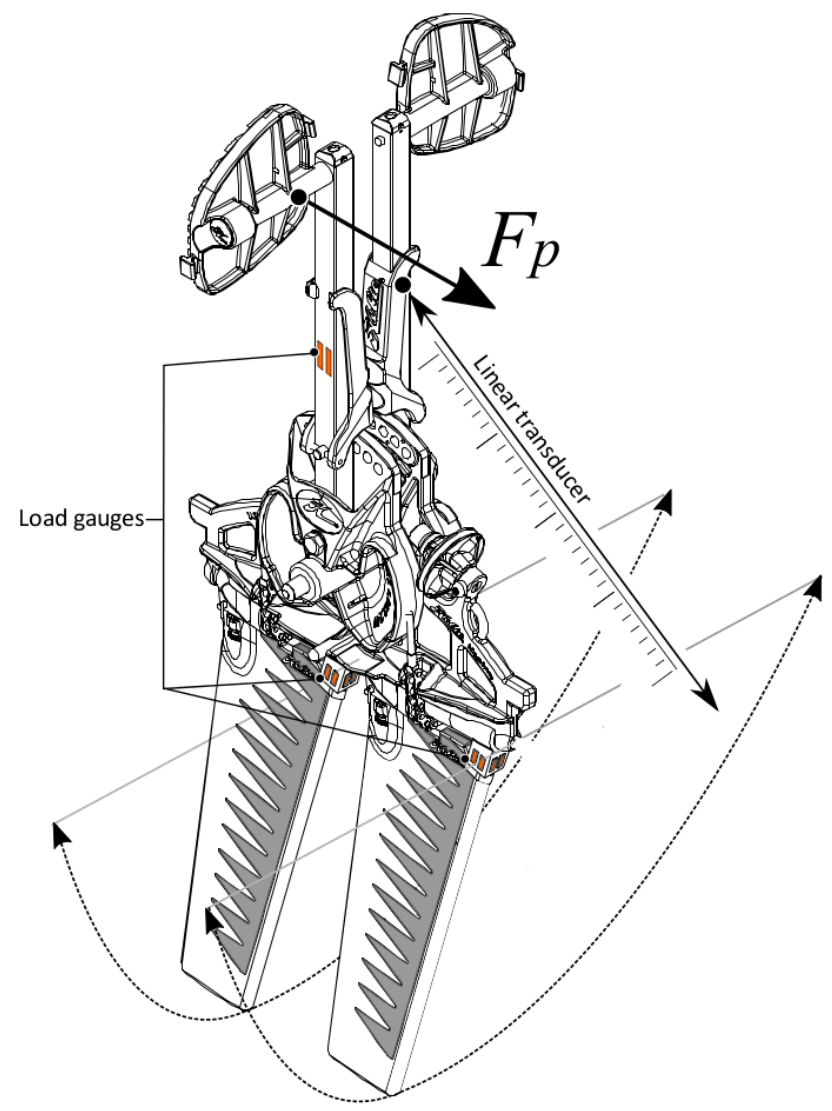

Figure 1: Mirage Drive propulsion system design and positioning of the gauges.

simultaneous measurement of the hydrodynamic loads on both the front and back foils.

\subsection{Mirage Drive propulsion system}

The Mirage Drive is a propulsion system that transforms pedaling motion of a driver into transverse sweeping motion of two underwater foils in a tandem configuration (see Figure 1). The system is installed by inserting it through the boat hull such that the foils protrude beneath the hull, and the pedals are accessible to the driver atop the boat (see Figure 2). Foils are placed in a tandem configuration and can sweep through a maximum angle of $196^{\circ}$. For the present setup the sweep amplitude is limited to $117^{\circ}$. As the driver pedals, the foils move under water. Periodic motion of each foil is dominated by rigid-body rotation of the main steel shaft (or mast) located at the foil's leading edge. Rigid body rotation takes place along the axis aligned 


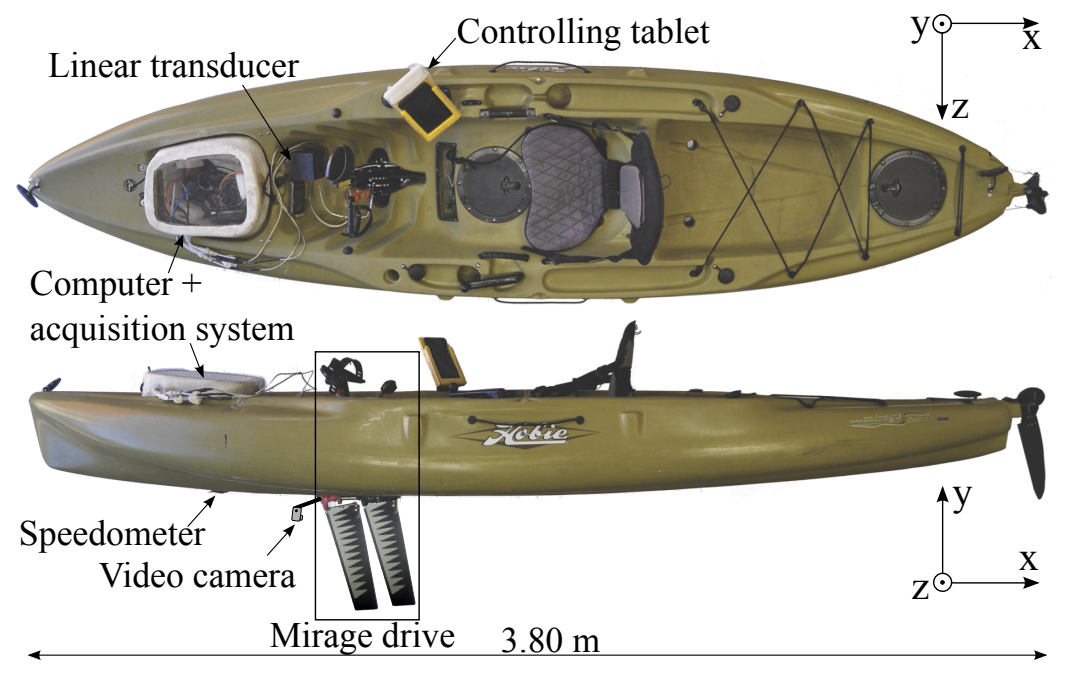

Figure 2: Instrumented kayak equipped with the Mirage Drive propulsion system.

with the vessel direction of travel. The compliant nature of the foils also results in twisting of the foil's axial cross-sections - as much as $40^{\circ}$ at the tip, see [6]) - which, in turn, generates the thrust force necessary to propel the vessel forward. During one stroke cycle the foils cross each other twice. The foils are $37 \mathrm{~cm}$ in length, and, at the instant of crossing, the closest clearance between them is less than $2.6 \mathrm{~cm}$. As a result, interaction between the foils is expected. The onboard instrumentation system developed in [6] for a single foil is updated in the present work to measure and characterize this interaction.

\subsection{Measurement system and data collected}

Full-scale measurements are performed on an Outback kayak (see Figure 2), also built by the Hobie Cat Co. The Outback is a $3.80 \mathrm{~m}, 40 \mathrm{~kg}$ fully rigged kayak with the Mirage Drive propulsion system installed. The kayak and propulsion system are equipped with dedicated instrumentation, which allows us to perform the following measurements. The field tests were performed in the San Diego Bay, and the second author was the kayak driver.

Hydrodynamic moments in the $x$ - and $z$-direction, denoted by $M_{x i}$ and $M_{z i}$, where $i=1$ and $i=2$ correspond to the front and back foil, respectively, are measured simultaneously using two $120 \mathrm{ohm}$ gauges (HBM LY11-3/120) mounted in a full Wheastone bridge configuration. The mast insert is replaced by a stainless steel square bar, where the gauges are placed. Figure 3 


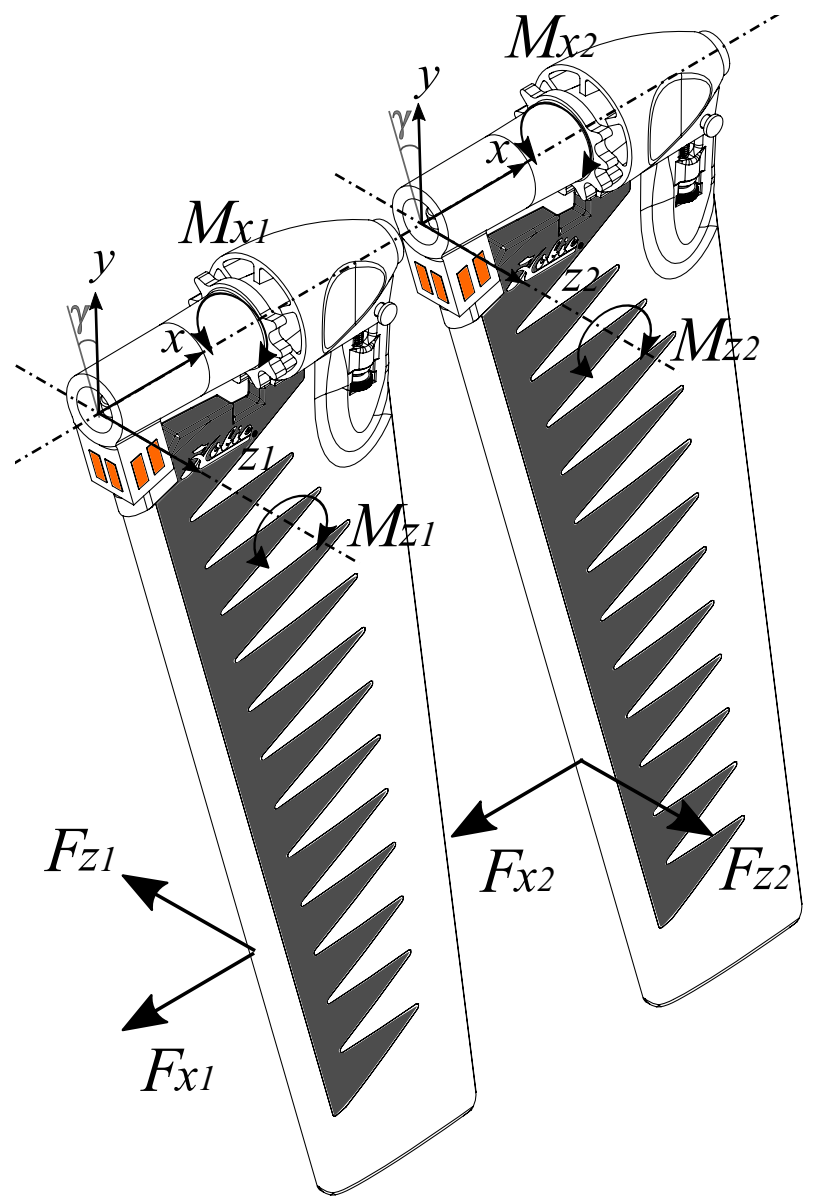

Figure 3: Coordinate systems employed in the tandem foil configuration. Gauges for hydrodynamic moment measurement are denoted by orange rectangles.

shows the gauges (denoted by orange rectangles) on each face of the square bar, as well as the coordinate frames used in the study. We assume that the $y$ - and $z$-axes rotate with each mast, while the $x$-axis is coincident with the kayak direction of travel (see Figure 2). Note that $M_{z i}$ is generated by the thrust force, while $M_{x i}$ arises due to the lateral forces acting on the foil. As a result, a good foil design may be characterized as having a larger $M_{z i}$ to- $M_{y i}$ ratio.

The left-pedal bracket is instrumented to measure the time-dependent force applied by the driver (see Figure 1). The load sensors are connected to a dedicated gauge analog amplifier and conditioner Expresso from HBM. The position of the pedal is measured by a linear transducer (see Figure 1) attached from the inside of the cockpit to the right bracket. The linear trans- 

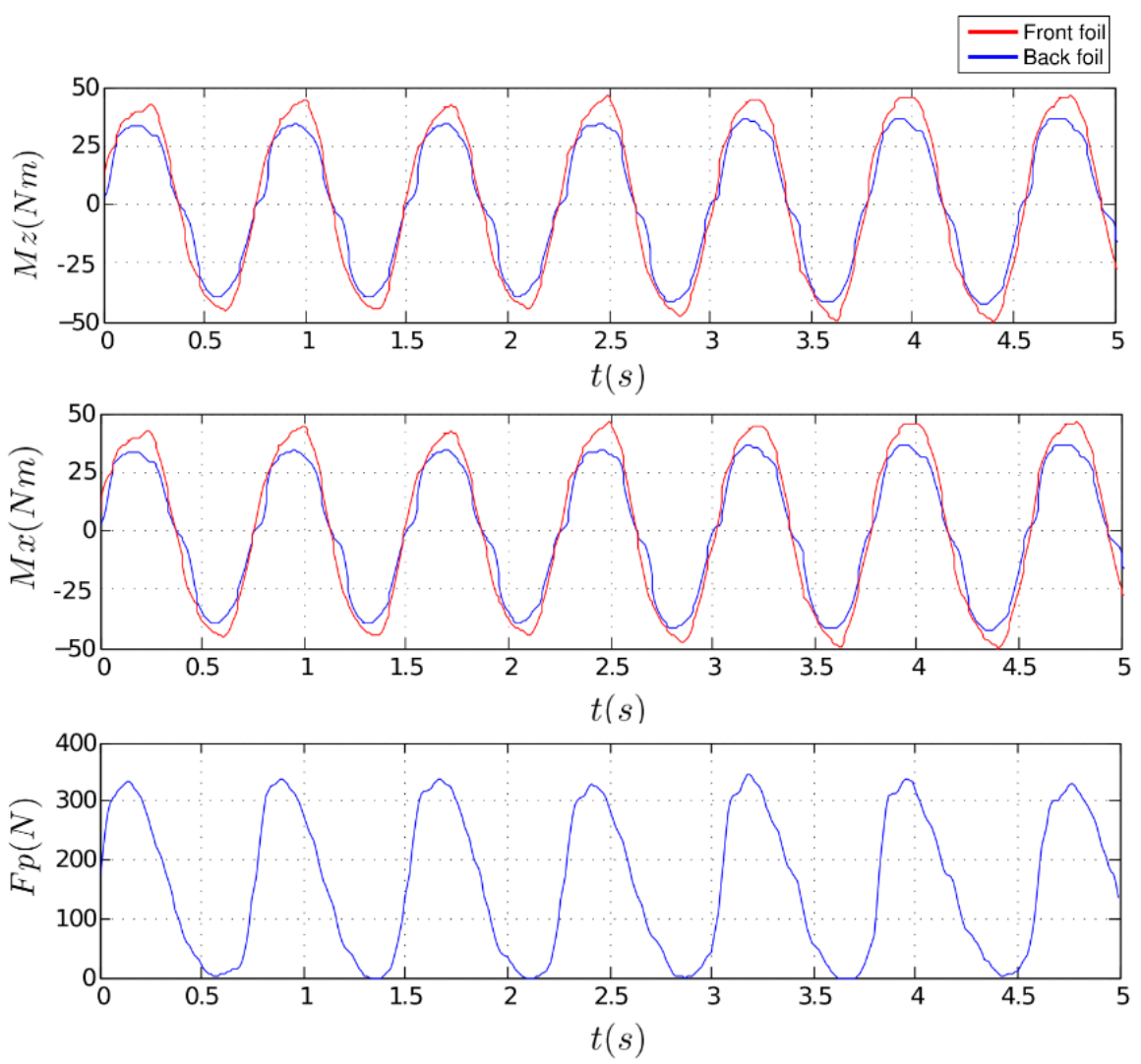

Figure 4: Time series of the moments $M_{x i}$ and $M_{z i}$ and the pedal-bracket load $F_{p}$ measured for a kayak speed of $2.1 \mathrm{~m} / \mathrm{s}$.

ducer is linked to a Dataq $430 \mathrm{AD}$ converter. The kayak speed is measured by a trough-the-hull speedometer installed on the kayak. The speed signal is recorded via a dedicated NMEA frame converter. Kayak speed is also recorded using a separate GPS device. The different instruments employed in the measurements are connected to a inboard PC, and synchronization of the heterogeneous data is done in postprocessing. The load sensors are calibrated to a precision error of $<1 \%$.

Figure 4 shows the times series of $M_{x i}$ and $M_{z i}$ and the pedal-bracket load $F_{p}$ measured for a kayak speed of $2.1 \mathrm{~m} / \mathrm{s}$. As expected, the propulsive moment $M_{z i}$ is non-negative and has double the frequency of the side moment $M_{x i}$. Because the kayak is propelled by a human driver, the measured data exhibits some differences from one period to the next. Nevertheless, one trend 
that clearly emerges is that moments acting on the back foil are generally greater than those acting on the front foil. This finding is novel and indicates that the interaction between the foils in the propulsion system considered in non-negligible.

\section{Governing Equations and Numerical Methods}

In this section we present the governing differential equation of the FSI problem in the strong form, and discuss their discretization choices that lead to an efficient, high-fidelity simulation framework for tandem hydrofoils.

\subsection{Equations for hydrodynamics and structural mechanics}

The hydrodynamics is governed by the Navier-Stokes equations for incompressible flows. The Navier-Stokes equations are posed on a moving spatial domain, and are written in the Arbitrary Lagrangian-Eulerian (ALE) frame [35] as follows:

$$
\begin{aligned}
& \rho_{1}\left(\left.\frac{\partial \mathbf{u}}{\partial t}\right|_{\hat{x}}+(\mathbf{u}-\hat{\mathbf{u}}) \cdot \boldsymbol{\nabla} \mathbf{u}-\mathbf{f}_{1}\right)-\boldsymbol{\nabla} \cdot \boldsymbol{\sigma}_{1}=\mathbf{0}, \\
& \boldsymbol{\nabla} \cdot \mathbf{u}=0
\end{aligned}
$$

where $\rho_{1}$ is the density, $\mathbf{u}$ is the velocity, $\mathbf{f}_{1}$ is the body force per unit mass, and $\hat{\mathbf{u}}$ is the velocity of fluid mechanics domain. The Cauchy stress, $\boldsymbol{\sigma}_{1}$, is given by

$$
\boldsymbol{\sigma}_{1}(\mathbf{u}, p)=-p \mathbf{I}+2 \mu \varepsilon(\mathbf{u})
$$

where $p$ is the pressure, $\mathbf{I}$ is the identity tensor, $\mu$ is the dynamic viscosity, and $\varepsilon(\mathbf{u})$ is the strain-rate tensor defined as

$$
\varepsilon(\mathbf{u})=\frac{\nabla \mathbf{u}+\nabla \mathbf{u}^{T}}{2}
$$

The time derivative in Eq. (1) is taken with respect to fixed referential domain coordinates $\hat{\mathbf{x}}$. All space derivatives are taken with respect to spatial coordinates of the current configuration $\mathbf{x}$.

The governing equations of structural mechanics, written in the Lagrangian frame [21], consist of the local balance of linear momentum and are given by

$$
\rho_{2}\left(\frac{\mathrm{d}^{2} \mathbf{y}}{\mathrm{d} t^{2}}-\mathbf{f}_{2}\right)-\boldsymbol{\nabla} \cdot \boldsymbol{\sigma}_{2}=\mathbf{0},
$$


where $\rho_{2}$ is the mass density, $\mathbf{f}_{2}$ is the body force per unit mass, $\boldsymbol{\sigma}_{2}$ is the Cauchy stress, and $\mathbf{y}$ is the unknown structural displacement.

At the fluid-structure interface, compatibility of the kinematics and tractions is enforced, namely,

$$
\begin{array}{r}
\mathbf{u}-\frac{\mathrm{d} \mathbf{y}}{\mathrm{d} t}=\mathbf{0}, \\
\boldsymbol{\sigma}_{1} \mathbf{n}_{1}+\boldsymbol{\sigma}_{2} \mathbf{n}_{2}=\mathbf{0},
\end{array}
$$

where $\mathbf{n}_{1}$ and $\mathbf{n}_{2}$ are the unit outward normal vectors to the fluid and structural mechanics domains, respectively. (Note that $\mathbf{n}_{1}=-\mathbf{n}_{2}$.)

The above constitutes a basic formulation of the FSI problem at the continuous level. In what follows, we discuss the discretization choices of the above governing equations that address the key challenges of, and leads to an efficient and robust approach for, the FSI simulation of hydrofoils in a tandem configuration. For a comprehensive discussion of discretization techniques, FSI coupling strategies, and application to a large class of problems in engineering the reader is referred to a recent FSI book [20].

\subsection{Discretization methods and FSI solution strategies}

For the hydrodynamics part of the FSI problem, the ALE-VMS method [12, 62] and weakly enforced essential boundary conditions $[15,17,18]$ are employed. The former is an extension of the residual-based variational multiscale (RBVMS) turbulence model [7] to moving domains using the ALE technique, while the latter relaxes boundary-layer resolution requirements to achieve good accuracy of fluid solution and loads prediction on meshes of reasonable size [1$3,28,29,40]$.

To simulate compliant hydrofoils in a tandem configuration and capture the interaction between the foils, the fluid domain is divided into two subdomains as shown in Figure 5. As the foils move in opposite directions, the corresponding subdomains rotate with them, creating a sliding interface. The compatibility conditions enforced at the sliding interface are

$$
\mathbf{u}_{\mathrm{F}}-\mathbf{u}_{\mathrm{B}}=\mathbf{0}
$$




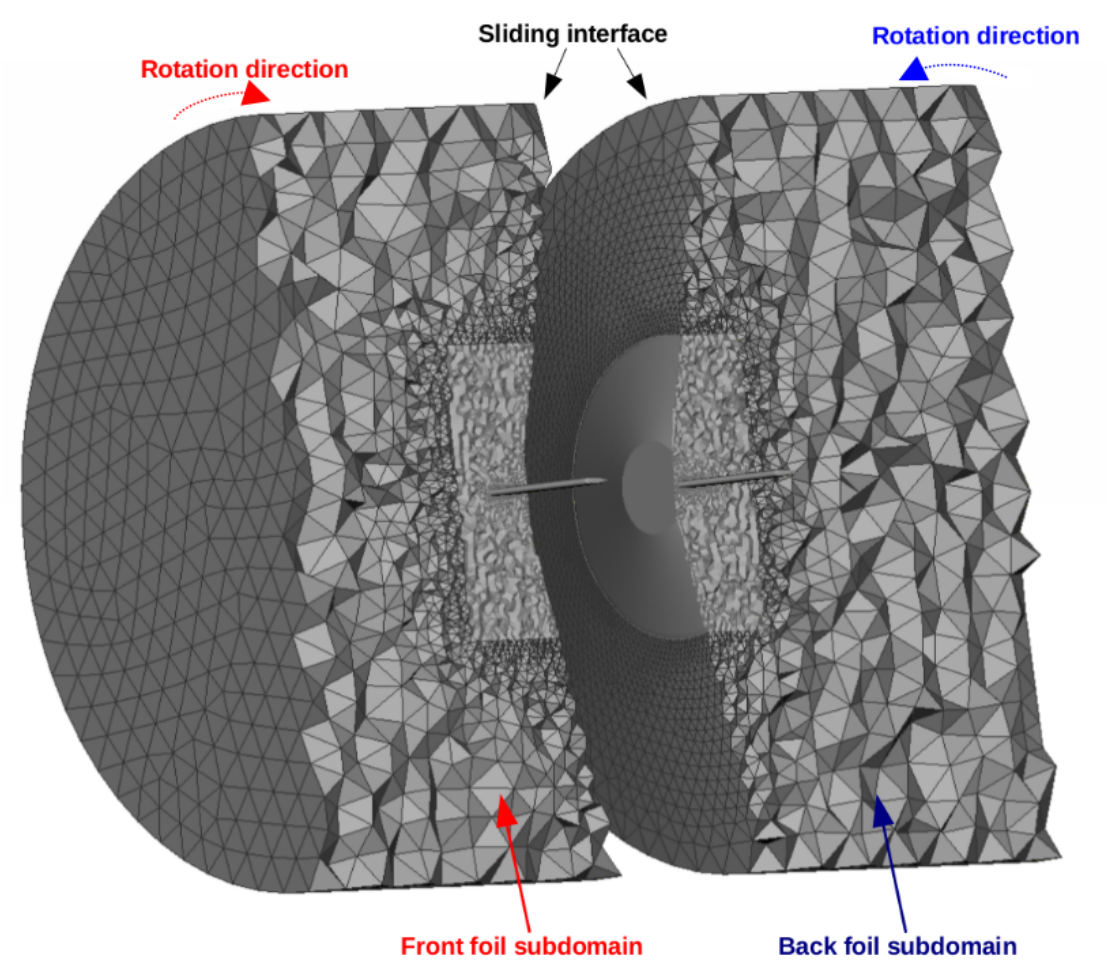

Figure 5: Fluid mechanics domain and mesh with a sliding interface shown. The front and back subdomains are artificially separated for illustration purposes.

and

$$
\left(-p_{\mathrm{F}} \mathbf{I}+2 \mu \varepsilon\left(\mathbf{u}_{\mathrm{F}}\right)\right) \mathbf{n}_{\mathrm{F}}+\left(-p_{\mathrm{B}} \mathbf{I}+2 \mu \varepsilon\left(\mathbf{u}_{\mathrm{B}}\right)\right) \mathbf{n}_{\mathrm{B}}=\mathbf{0},
$$

where all quantities with subscripts ' $\mathrm{F}$ ' and ' $\mathrm{B}$ ' refer to the front and back foil subdomains, respectively, and $\mathbf{n}_{\mathrm{F}}$ and $\mathbf{n}_{\mathrm{B}}$ are the corresponding normal vectors. Compatibility conditions given by the above Eqs. (8) and (9) are enforced weakly in the framework of the sliding-interface technique $[16,31]$. The ALE-VMS and sliding interface formulations are discretized using linear FEM.

The structural mechanics of compliant hydrofoils is modeled using a combination of the Kirchhoff-Love shell [41, 42] and beam/cable [53] formulations. Both are discretized using IGA based on non-uniform rational B-spline (NURBS) [24, 34] and make use of only displacement degrees of freedom. Using IGA for foil modeling presents a good combination of 
efficiency, since no rotational degrees-of-freedom are employed, accuracy, since NURBS are a higher-order accurate discretization technique [9], and robustness. The latter refers to the fact that higher-order continuity of NURBS induces smooth deformation of the structural surface, which in turn, translates to smooth deformation of the fluid mechanics mesh at the fluidstructure interface, and, as a result, leads to better quality of boundary-layer discretization near moving surfaces.

The coupled FSI problem is formulated using an augmented Lagrangian approach for FSI, which was originally proposed in [11] to handle boundary-fitted mesh computations with nonmatching fluidstructure interface discretizations. The key feature of the method is formal elimination of the Lagrange multiplier variable, which results in weak enforcement of the fluidstructure interface compatibility conditions using only primal variables (i.e., fluid velocity and pressure, and structure displacement), and, as a consequence, leads to increased efficiency compared to classical Lagrange multiplier methods.

To accommodate the global rotational motion of the foils with superposed local elastic deformation, and to maintain a moving-mesh discretization with good-quality boundary-layer resolution critical for hydrodynamics accuracy, the fluid domain mesh is moved as follows. While at the fluid-structure interface the fluid mechanics mesh follows the motion of the foils, the outer boundaries of the foil subdomains are restricted to only undergo rigid rotation. This choice of domain motion preserves the geometry of the sliding interface. The same rigid-body motion is applied at the leading edge of each foil. The rest of the mesh motion is obtained by solving the equations of elastostatics with Jacobian-based stiffening [37, 59, 85-88].

The generalized- $\alpha$ method $[8,22,36]$ is employed to advance to FSI equations in time. Quasi-direct coupling [20] is employed to solve the resulting coupled nonlinear equation system. In the quasi-direct coupling technique, at every Newton-Raphson iteration, solution increments of the fluid and structural mechanics subproblems are computed simultaneously. Linearization of the coupled FSI equation system takes into account not only the individual fluid and structural mechanics subproblems, but also compatibility conditions at the fluid-structure and sliding interfaces. Although this approach completely circumvents convergence difficulties associated with high added mass and, as a result, enables robust simulation of hydrofoil FSI, its 
efficient numerical implementation is more challenging compared to its block-iterative counterpart. In the present work, for the fluid-structure equation block, we make use of a matrix-free implementation of the flexible generalized minimum residual (GMRES) $[55,56]$ technique preconditioned with individual fluid and structure linearized problems. The matrix-free technique enables relatively straightforward treatment of nonmatching interfaces, while preconditioning reduces the number of GMRES iterations required for convergence in order to increase the overall efficiency. For a comprehensive discussion of matrix-free and preconditioning techniques in FSI applications the reader is referred to [20].

\section{Compliant Hydrofoil Simulation}

\subsection{Computational setup}

\begin{tabular}{ccc}
\hline Material & Black & Grey \\
\hline Young's Modulus (MPa) & 27.58 & 12.24 \\
\hline Poisson's Ratio & 0.47 & 0.47 \\
\hline Density $\left(\mathrm{kg} / \mathrm{m}^{3}\right)$ & 1200 & 1200 \\
\hline
\end{tabular}

Table 1: Material properties of the foil.

In present simulation, the front and back foils are identical in terms of geometry and material properties, taken from a single-foil simulation reported in [6]. The foils are made of a solid rubber-like material and have a symmetric shape. In [6], an equivalent IGA Kirchhoff-Love shell model of the foil was constructed and validated by reproducing the deflection and twistangle data of the structural "sag test" devised for that purpose. The foil mesh is comprised of multiple NURBS patches and has nearly 10,000 quadratic elements (see Figure 6). The bending strip technique [41] is employed to deal with the multiple-patch discretization. The structure has two material zones, referred to as Black and Grey material, as shown on Figures 1 and 3. Each zone is made of an isotropic St. Venant-Kirchhoff material with properties summarized in Table 1. (See [43] for a general hyperelastic modeling framework for Kirchhoff-Love shells.) The zigzag pattern of the material is designed such that the foil has the desired flexibility and stiffness. 


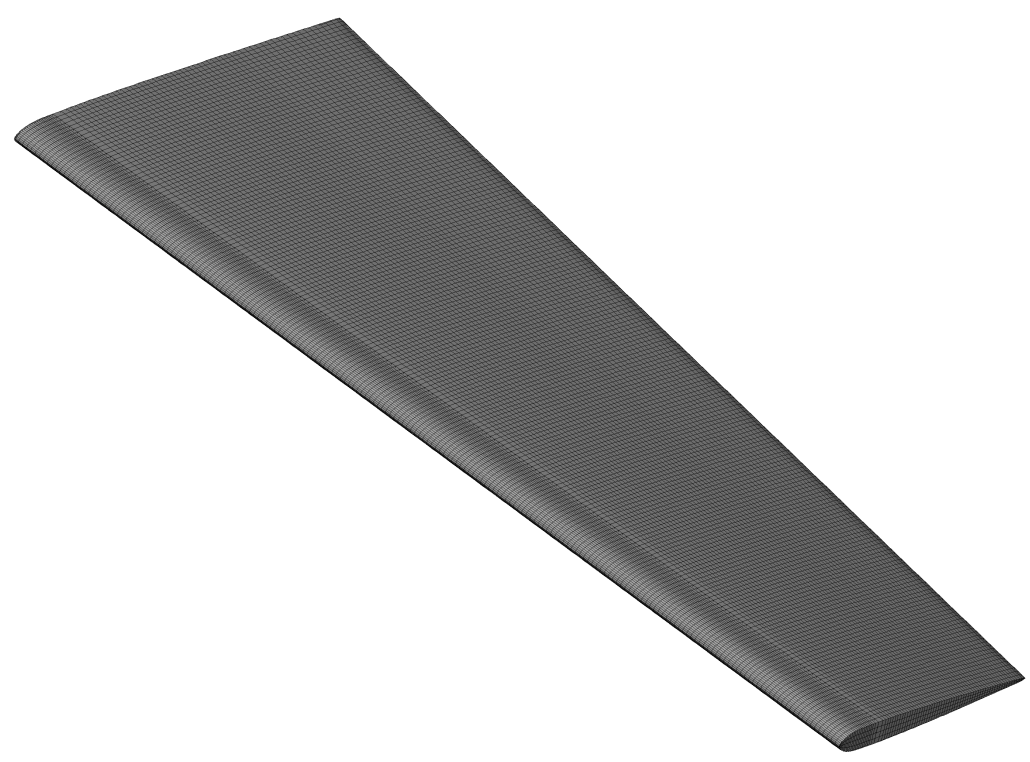

Figure 6: NURBS mesh of the foil shell model comprised of nearly 10,000 quadratic NURBS elements.

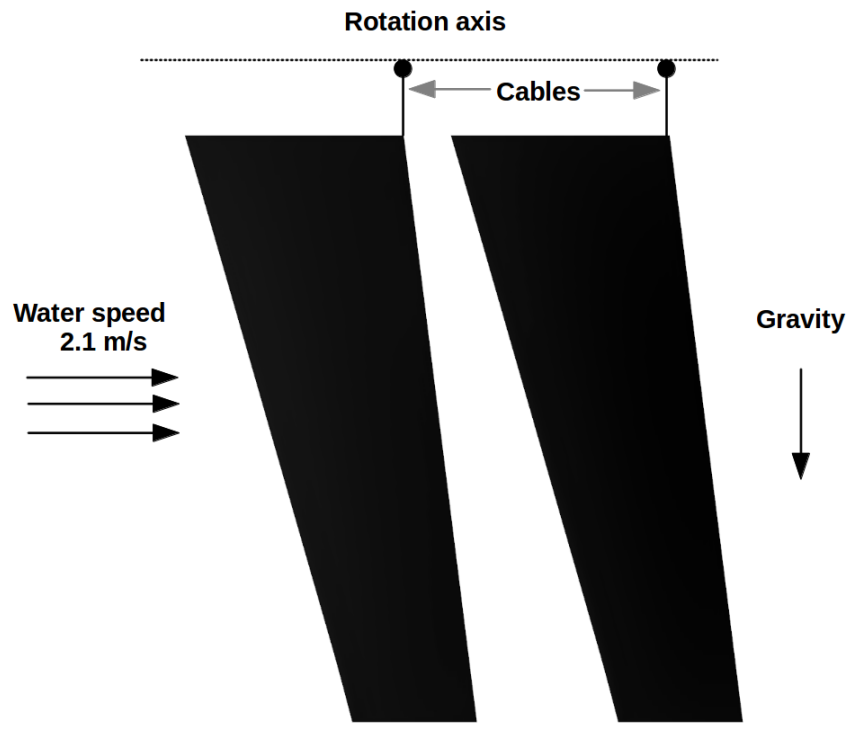

Figure 7: Side view of the tandem foil configuration.

The foil tandem configuration is shown in Figure 7 and the problem setup is shown in Figure 8. The foils are $0.37 \mathrm{~m}$ in length, and clearance between them is $0.0259 \mathrm{~m}$ at the top and $0.0772 \mathrm{~m}$ at the bottom axial cross-sections. The distance between the rotation axis and 


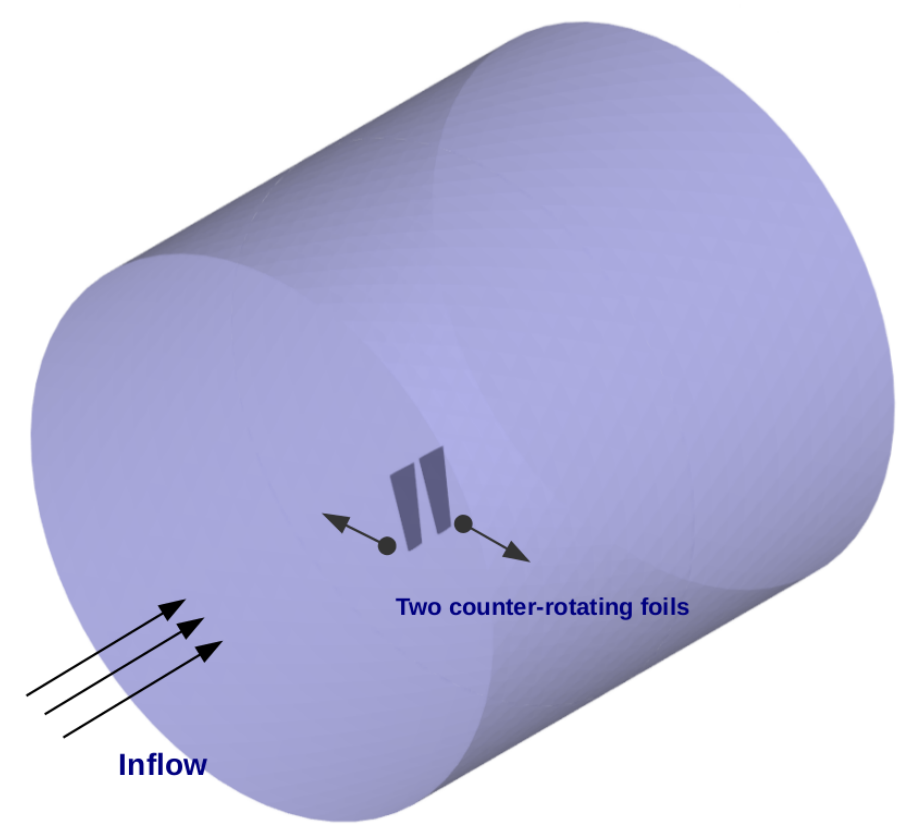

Figure 8: Computational domain and problem setup.

top surface of the foils is $0.047 \mathrm{~m}$. The simulation is performed at prescribed steady inlet water speed of $2.1 \mathrm{~m} / \mathrm{s}$. To drive the system, time-dependent rotation boundary conditions are applied on the leading edge of each foil. Rotation angles for the front and back foils are given by

$$
\theta_{\mathrm{F}}(t)=\frac{A \pi}{2} \sin \left(\frac{2 \pi t}{T}\right)
$$

and

$$
\theta_{\mathrm{B}}(t)=-\frac{A \pi}{2} \sin \left(\frac{2 \pi t}{T}\right),
$$

respectively. In the above equations $\frac{A \pi}{2}$ and $T$ give the maximum rotation angle and stroke period, respectively. In the present simulation, $A=0.6501$ and $T=0.6821 \mathrm{~s}$, which are typical conditions for high-speed cruising, and which are also consistent with the field test results presented earlier.

The connection between the foil top-cross-section trailing edge and rotation axis is modeled using isogeometric cable structures [53]. The cables are loosened by giving them a slightly 


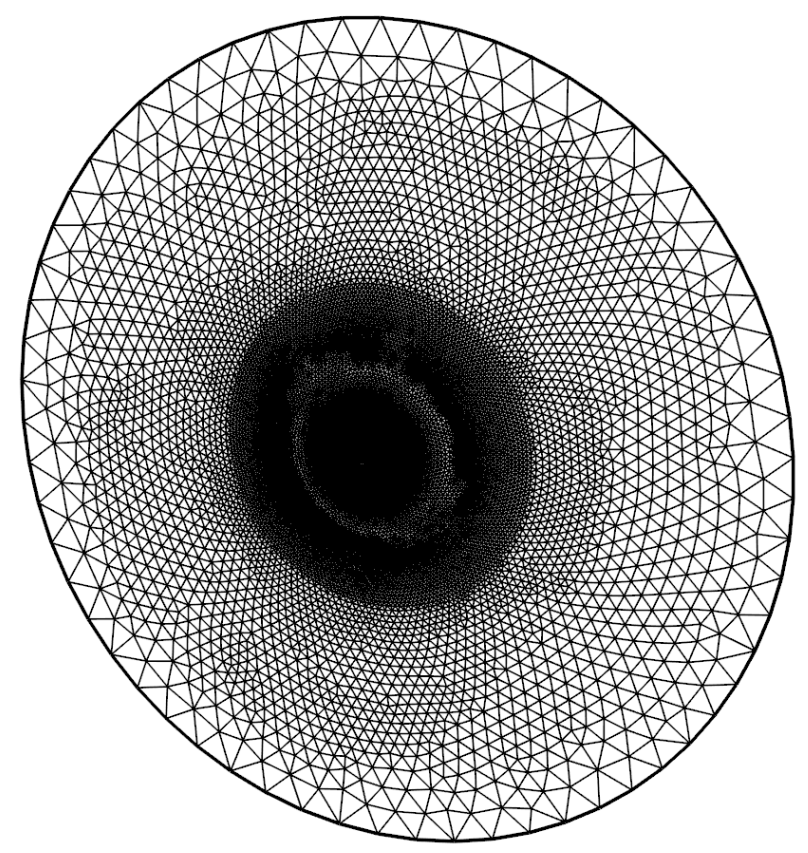

Figure 9: Triangular mesh of the sliding interface.

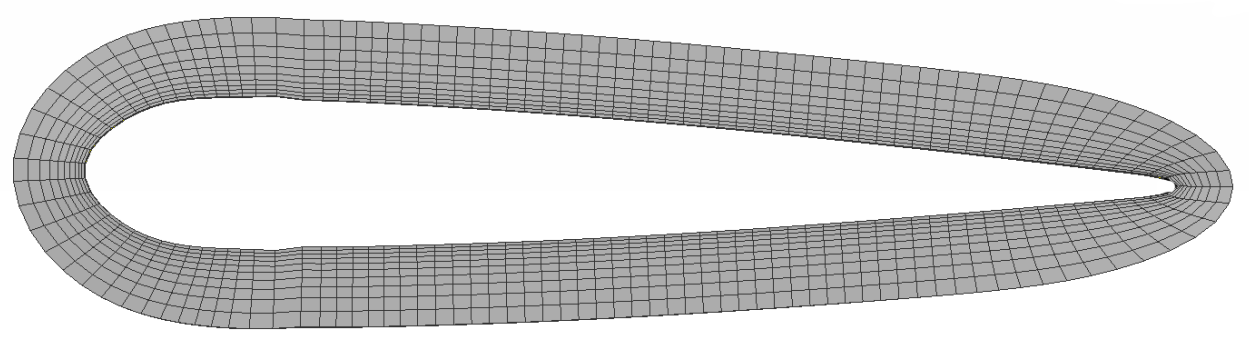

Figure 10: Triangular-prism discretization of the foil boundary layers.

curved initial-configuration profile. This setup mimics the actual attachment mechanism of the foil trailing edge to the rotation axis, and allows the foils to develop higher twisting angles needed for efficient propulsion. Each cable has one end attached to the rotation axis and the other to the trailing edge of the foil top cross-section (see Figure 7). The cables are discretized using a single quadratic NURBS element. We note that cable elements are employed with the sole purpose to appropriately constrain the foil trailing-edge motion. Cable elements do not receive forces from the fluid, nor do they affect the fluid kinematics. 


\begin{tabular}{ccc}
\hline & Num. of nodes & Num. of elements \\
\hline Mesh & 550,557 & $2,224,857$ \\
\hline
\end{tabular}

Table 2: Fluid mechanics mesh statistics.

The fluid mechanics domain and mesh are designed as follows. The domain boundary is a cylinder with radius of $0.96 \mathrm{~m}$ and length of $1.5 \mathrm{~m}$. A refined cylinder is built around the foils in order to better capture the turbulence generated by the foils. As shown in Figure 5, the domain is divided into two subdomains separated by a sliding interface. We choose a cone-shaped sliding interface, which makes the trailing edge of the front foil and leading edge of the back foil nearly equidistant from the interface. The sliding-interface mesh is shown in Figure 9. The elements are clustered toward the cone center to have a more accurate representation of the interaction between the foils. The mesh gradually coarsens toward the lateral boundaries where the flow is uniform. The volume mesh makes use of triangular prisms in the foil boundary layers (see Figure 10), and tetrahedra elsewhere. The mesh statistics are summarized in Table 2.

Uniform inflow velocity is imposed strongly at the inlet. No-penetration and zero streamwisetraction boundary conditions are applied on the cylinder lateral surfaces. Zero traction boundary conditions are imposed at the outlet. The computation is performed in a parallel computing environment using 144 processors. The fluid mesh is partitioned into subdomains using METIS [39], and each subdomain is assigned to a compute core. The parallel implementation methodology employed may be found in [27]. The time step is set to $1.5 \times 10^{-4} \mathrm{~s}$, and the simulation is performed for two stroke cycles.

\subsection{Results}

Time histories of the computed and measured hydrodynamic moments $M_{x i}$ and $M_{z i}$ are plotted in Figure 11. Good agreement both in the hydrodynamic moment magnitude as well as other trends in the moment time history is achieved between the computational results and field test data. When the foils are separated by a large distance, they barely feel each other's presence, and the hydrodynamic moments acting on the foils are nearly identical. However, when the foils cross each other, strong interaction between them can be observed, especially in the moment curves coming from the FSI simulation. One important trend that is observed experimentally 

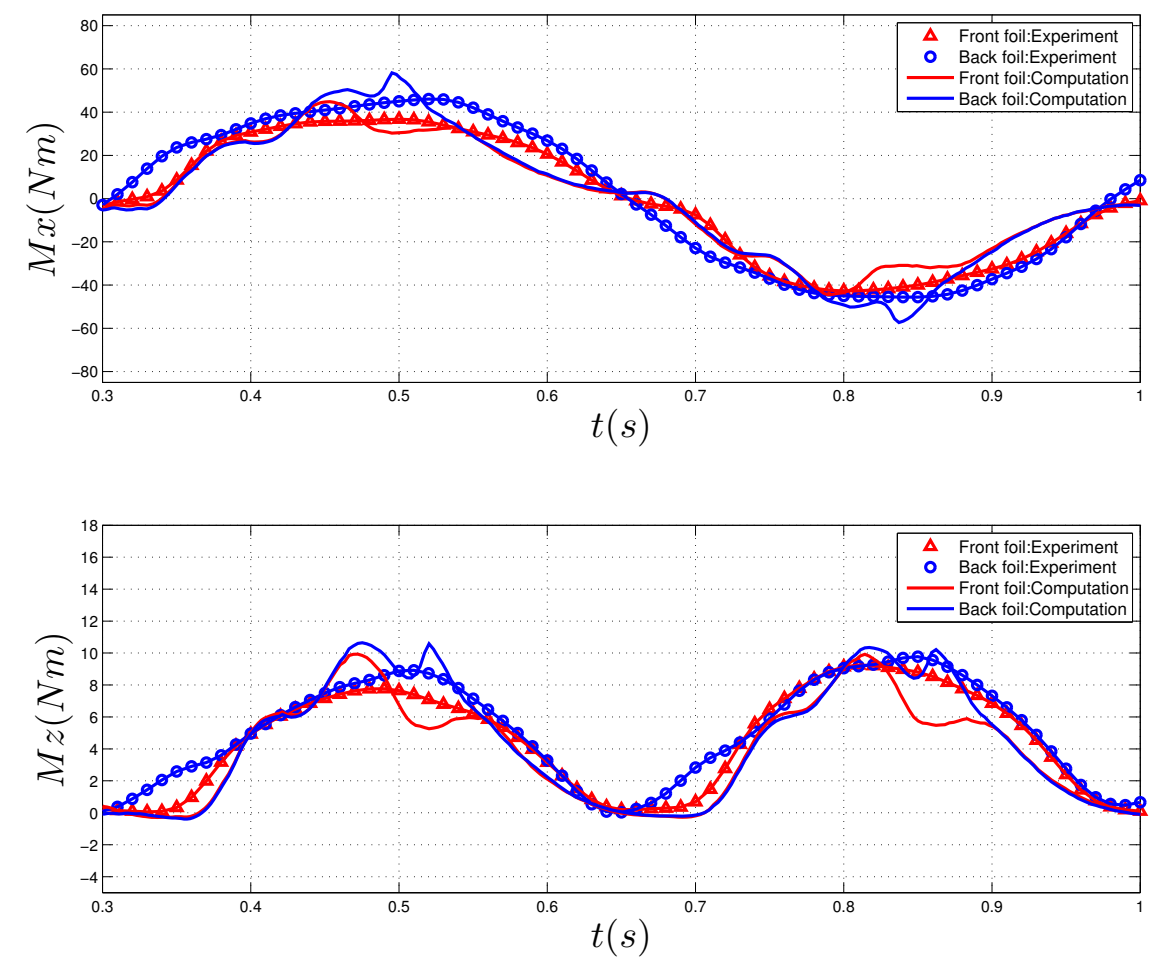

Figure 11: Time history of hydrodynamic moments $M_{x i}$ and $M_{z i}$. Both experimental and computational results are plotted for comparison.

and reproduced in the computation is that during the entire stroke cycle moments acting on the back foil are greater than or equal to those acting on the front foil.

Time histories of the twist angle at four different cross sections along the foil axis are plotted in Figure 12. The cross-section locations are indicated in Figure 13. The maximum twist angle during the stroke cycle is in excellent agreement with the experimental data reported in [6]. Although fairly significant differences in the hydrodynamic moments were observed between the front and back foils, this is not the case for the twist angle. For both foils time histories of the twist angle are very similar, with difference on the order of $1^{\circ}$ observed right after the foils cross each other. The difference in the twist angle becomes more pronounced closer to the foil tip where the maximum twist occurs. The curves suggest that the back foil twists more than the front one, which is consistent with the moment curve trends discussed in the previous 


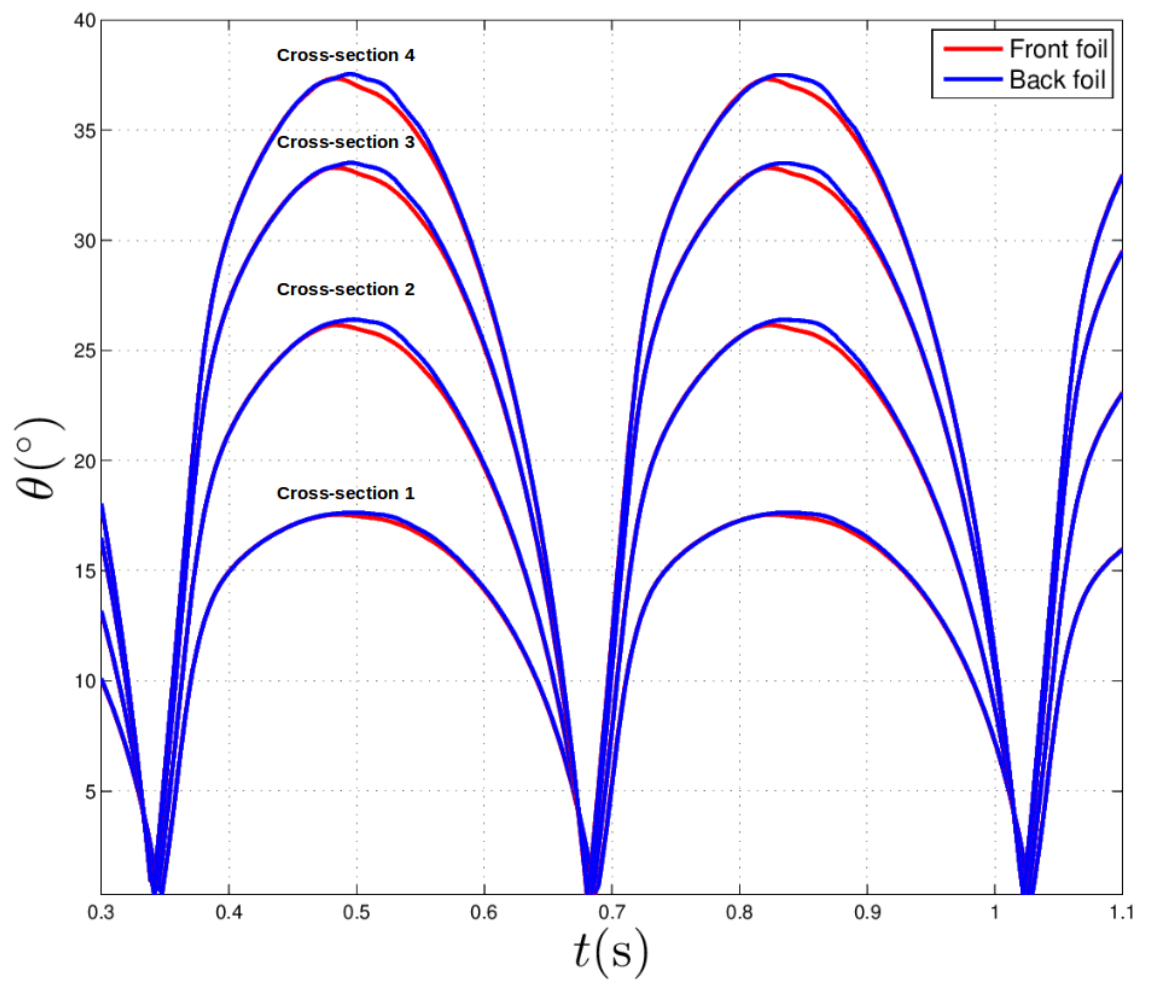

Figure 12: Time history of twist angle at four cross sections.

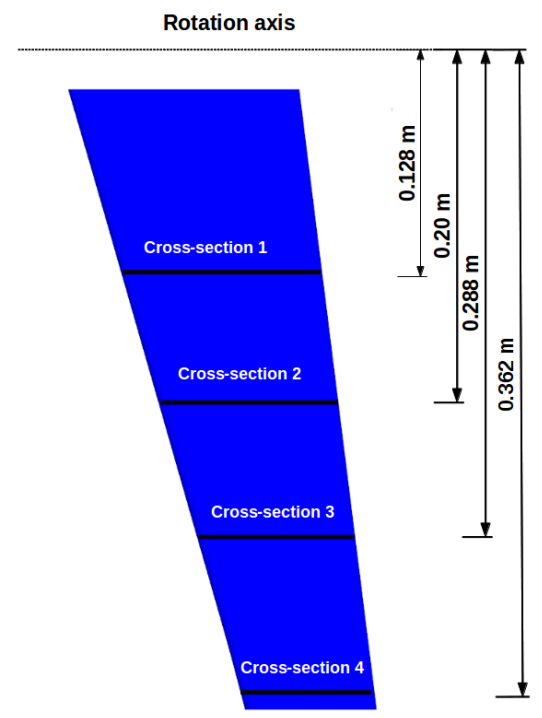

Figure 13: Locations of four cross sections. 

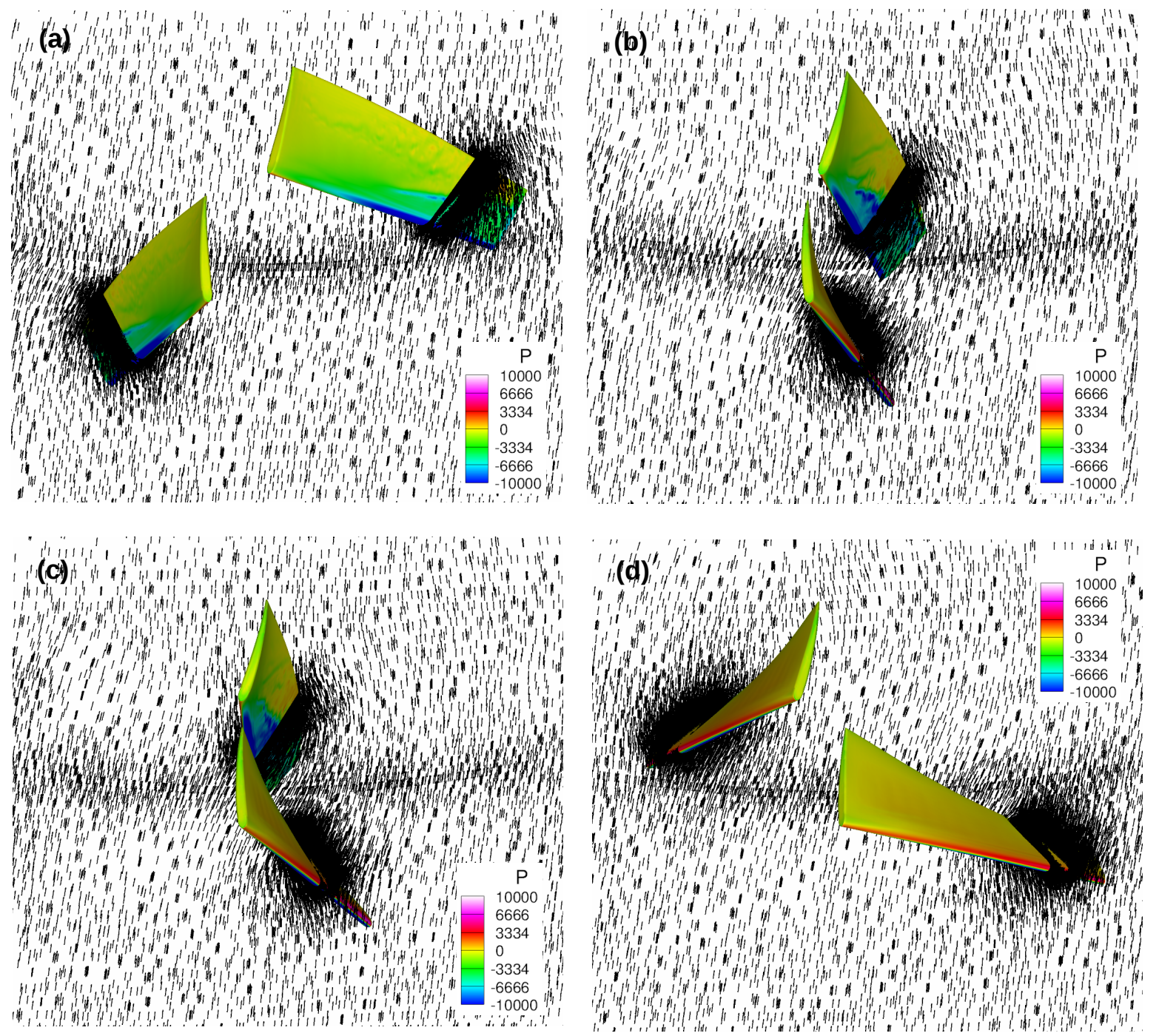

Figure 14: Fluid velocity vectors on a cut plane superposed on foils in deformed configuration colored by fluid pressure (in $\mathrm{Pa}$ ).

paragraph. The higher twist of the back foil decreases the flow angle of incidence leading to slight enhancement of its propulsion efficiency.

Figure 14 shows time instances of the fluid velocity vectors on a cut plane superposed on foils in deformed configuration and colored by fluid pressure. These correspond to instances before, during, and after the crossing event. Changes in the flow velocity patterns as the foils get closer to one another are clearly visible. Significant twisting of the foils may also be observed 
(a)

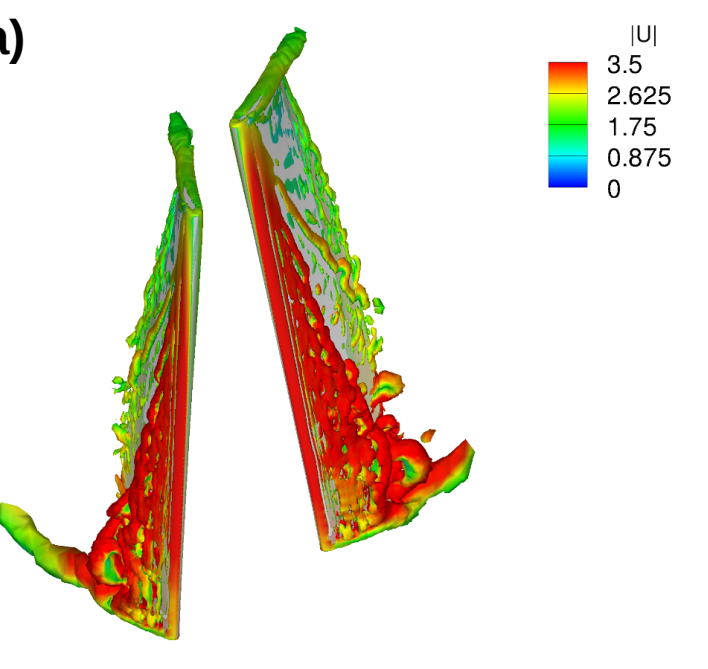

(c)

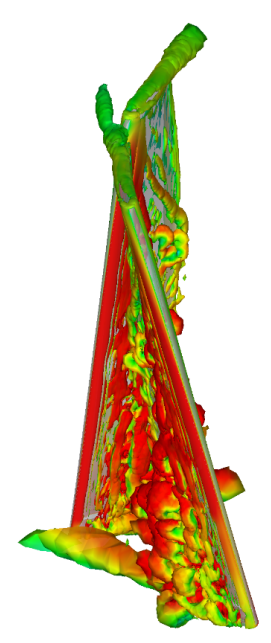

(b)

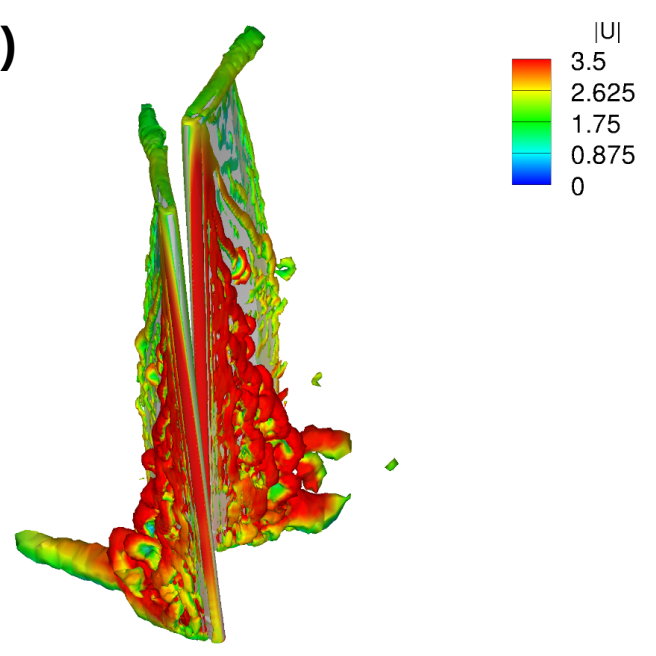

(d)

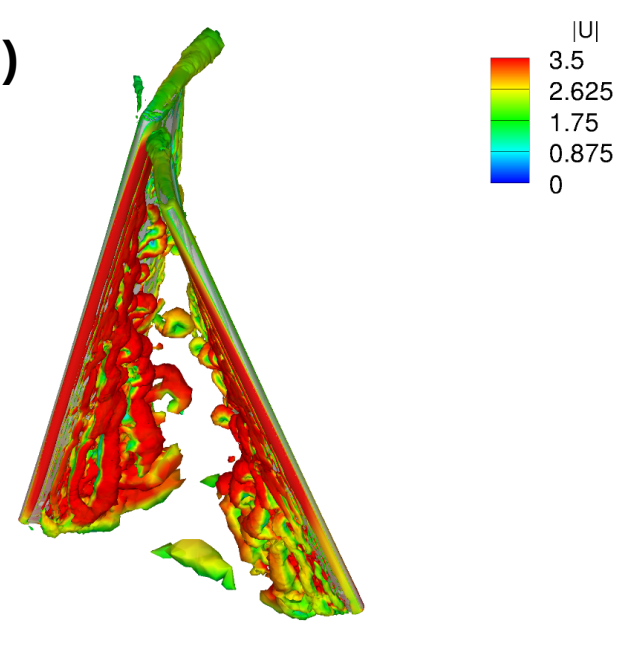

Figure 15: Vorticity isosurfaces colored by flow speed (in $\mathrm{m} / \mathrm{s}$ ).

in the figure. Figure 15 shows time instances of vorticity isosurfaces colored by flow speed, also before, during, and after the crossing event. Significant vorticity is generated on the suction side of the foil accompanied by massive flow separation. On the pressure side the the flow appears to be attached and very little vorticity is present. The complexity of the underlying wall-bounded turbulent flow is also clearly seen in the figure, which underscores the necessity to use advanced FSI simulation techniques for this problem class. 


\section{Conclusions}

It behooves a successful computational FSI framework for compliant-hydrofoil-based propulsion to incorporate accurate boundary-layer discretization near the foil surfaces, to accommodate the foil relative motions that periodically bring them in close proximity to one another, and to employ a robust FSI coupling strategy that is insensitive to the high added mass present in the application. Such a framework was presented in the current paper and successfully employed in the FSI simulation of the Mirage Drive propulsion system using two oscillating compliant hydrofoils in a tandem configuration. The simulations were able to accurately predict the hydrodynamic loads and details of foil deformation as compared to the measured data from field tests, which are also described in the present paper. Furthermore, the FSI simulation was able to capture the main trends in the interaction between the two foils, such as stronger interaction dynamics during foil crossing and larger hydrodynamic moments acting on the back foil compared to the front foil.

\section{Acknowledgements}

This work was partially supported through the NSF CAREER Award No. 1055091. This support is gratefully acknowledged.

\section{References}

[1] I. Akkerman, Y. Bazilevs, D. J. Benson, M. W. Farthing, and C. E. Kees. Free-surface flow and fluid-object interaction modeling with emphasis on ship hydrodynamics. Journal of Applied Mechanics, 79:010905, 2012.

[2] I. Akkerman, Y. Bazilevs, C. E. Kees, and M. W. Farthing. Isogeometric analysis of freesurface flow. Journal of Computational Physics, 230:4137-4152, 2011.

[3] I. Akkerman, J. Dunaway, J. Kvandal, J. Spinks, and Y. Bazilevs. Toward free-surface modeling of planing vessels: simulation of the Fridsma hull using ALE-VMS. Computational Mechanics, 50:719-727, 2012. 
[4] B. Augier, P. Bot, F. Hauville, and M. Durand. Experimental validation of unsteady models for fluid structure interaction: Application to yacht sails and rigs. Journal of Wind Engineering and Industrial Aerodynamics, 101:53 - 66, 2012.

[5] B. Augier, P. Bot, F. Hauville, and M. Durand. Dynamic behavior of a flexible yacht sail plan. Ocean Engineering, 66:32-43, 2013.

[6] B. Augier, J. Yan, A. Korobenko, J. Czarnowski, G. Ketterman, and Y. Bazilevs. Experimental and numerical FSI study of compliant hydrofoils. Computational Mechanics, 55:1079-1090, 2015.

[7] Y. Bazilevs, V. M. Calo, J. A. Cottrel, T. J. R. Hughes, A. Reali, and G. Scovazzi. Variational multiscale residual-based turbulence modeling for large eddy simulation of incompressible flows. Computer Methods in Applied Mechanics and Engineering, 197:173-201, 2007.

[8] Y. Bazilevs, V. M. Calo, T. J. R. Hughes, and Y. Zhang. Isogeometric fluid-structure interaction: theory, algorithms, and computations. Computational Mechanics, 43:3-37, 2008.

[9] Y. Bazilevs, L. Beirao da Veiga, J. A. Cottrell, T. J. R. Hughes, and G. Sangalli. Isogeometric analysis: Approximation, stability and error estimates for $h$-refined meshes. Mathematical Models and Methods in Applied Sciences, 16:1031-1090, 2006.

[10] Y. Bazilevs, M.-C. Hsu, D. Benson, S. Sankaran, and A. Marsden. Computational fluidstructure interaction: Methods and application to a total cavopulmonary connection. Computational Mechanics, 45:77-89, 2009.

[11] Y. Bazilevs, M.-C. Hsu, and M. A. Scott. Isogeometric fluid-structure interaction analysis with emphasis on non-matching discretizations, and with application to wind turbines. Computer Methods in Applied Mechanics and Engineering, 249-252:28-41, 2012.

[12] Y. Bazilevs, M.-C. Hsu, K. Takizawa, and T. E. Tezduyar. ALE-VMS and ST-VMS methods for computer modeling of wind-turbine rotor aerodynamics and fluid-structure interaction. Mathematical Models and Methods in Applied Sciences, 22(supp02):1230002, 2012. 
[13] Y. Bazilevs, M.-C. Hsu, Y. Zhang, W. Wang, T. Kvamsdal, S. Hentschel, and J. Isaksen. Computational fluid-structure interaction: Methods and application to cerebral aneurysms. Biomechanics and Modeling in Mechanobiology, 9:481-498, 2010.

[14] Y. Bazilevs, M.-C. Hsu, Y. Zhang, W. Wang, X. Liang, T. Kvamsdal, R. Brekken, and J. Isaksen. A fully-coupled fluid-structure interaction simulation of cerebral aneurysms. Computational Mechanics, 46:3-16, 2010.

[15] Y. Bazilevs and T. J. R. Hughes. Weak imposition of Dirichlet boundary conditions in fluid mechanics. Computers and Fluids, 36:12-26, 2007.

[16] Y. Bazilevs and T. J. R. Hughes. NURBS-based isogeometric analysis for the computation of flows about rotating components. Computational Mechanics, 43:143-150, 2008.

[17] Y. Bazilevs, C. Michler, V. M. Calo, and T. J. R. Hughes. Weak Dirichlet boundary conditions for wall-bounded turbulent flows. Computer Methods in Applied Mechanics and Engineering, 196:4853-4862, 2007.

[18] Y. Bazilevs, C. Michler, V. M. Calo, and T. J. R. Hughes. Isogeometric variational multiscale modeling of wall-bounded turbulent flows with weakly enforced boundary conditions on unstretched meshes. Computer Methods in Applied Mechanics and Engineering, 199:780-790, 2010.

[19] Y. Bazilevs, K. Takizawa, and T. E. Tezduyar. Challenges and directions in computational fluid-structure interaction. Mathematical Models and Methods in Applied Sciences, 23:215-221, 2013.

[20] Y. Bazilevs, K. Takizawa, and T. E. Tezduyar. Computational Fluid-Structure Interaction: Methods and Applications. Wiley, 2013.

[21] T. Belytschko, W. K. Liu, and B. Moran. Nonlinear Finite Elements for Continua and Structures. Wiley, 2000.

[22] J. Chung and G. M. Hulbert. A time integration algorithm for structural dynamics withimproved numerical dissipation: The generalized- $\alpha$ method. Journal of Applied Mechanics, 60:371-75, 1993.

[23] S.A. Combes and T.L. Daniel. Shape, flapping and flexion: wing and fin design for forward flight. Journal of Experimental Biology, 204(12):2073-2085, 2001. 
[24] J. A. Cottrell, T. J. R. Hughes, and Y. Bazilevs. Isogeometric Analysis: Toward Integration of CAD and FEA. Wiley, Chichester, 2009.

[25] J. O. Dabiri. Potential order-of-magnitude enhancement of wind farm power density via counter-rotating vertical-axis wind turbine arrays. Journal of Renewable and Sustainable Energy, 3:043104, 2011.

[26] F.E. Fish. Performance constraints on the maneuverability of flexible and rigid biological systems. In International Synposium on Unmanned Unthethered Submersible Technology, pages 394-406. University of New Hampshire-Marine Systems, 1999.

[27] M.-C. Hsu, I. Akkerman, and Y. Bazilevs. High-performance computing of wind turbine aerodynamics using isogeometric analysis. Computers $\mathcal{E}$ Fluids, 49:93-100, 2011.

[28] M.-C. Hsu, I. Akkerman, and Y. Bazilevs. Wind turbine aerodynamics using ALE-VMS: Validation and the role of weakly enforced boundary conditions. Computational Mechanics, 50:499-511, 2012. doi:10.1007/s00466-012-0686-x.

[29] M.-C. Hsu, I. Akkerman, and Y. Bazilevs. Finite element simulation of wind turbine aerodynamics: Validation study using NREL Phase VI experiment. Wind Energy, 17:461$481,2014$.

[30] M.-C. Hsu and Y. Bazilevs. Blood vessel tissue prestress modeling for vascular fluidstructure interaction simulations. Finite Elements in Analysis and Design, 47:593-599, 2011.

[31] M.-C. Hsu and Y. Bazilevs. Fluid-structure interaction modeling of wind turbines: simulating the full machine. Computational Mechanics, 50:821-833, 2012.

[32] M.-C. Hsu, D. Kamensky, Y. Bazilevs, M. S. Sacks, and T. J. R. Hughes. Fluid-structure interaction analysis of bioprosthetic heart valves: significance of arterial wall deformation. Computational Mechanics, 54:1055-1071, 2014.

[33] M.-C. Hsu, D. Kamensky, F. Xu, J. Kiendl, C. Wang, M. C. H. Wu, J. Mineroff, A. Reali, Y. Bazilevs, and M. S. Sacks. Dynamic and fluid-structure interaction simulations of bioprosthetic heart valves using parametric design with T-splines and Fung-type material models. Computational Mechanics, 55:1211-1225, 2015. 
[34] T. J. R. Hughes, J. A. Cottrell, and Y. Bazilevs. Isogeometric analysis: CAD, finite elements, NURBS, exact geometry, and mesh refinement. Computer Methods in Applied Mechanics and Engineering, 194:4135-4195, 2005.

[35] T. J. R. Hughes, W. K. Liu, and T. K. Zimmermann. Lagrangian-Eulerian finite element formulation for incompressible viscous flows. Computer Methods in Applied Mechanics and Engineering, 29:329-349, 1981.

[36] K. E. Jansen, C. H. Whiting, and G. M. Hulbert. A generalized- $\alpha$ method for integrating the filtered Navier-Stokes equations with a stabilized finite element method. Computer Methods in Applied Mechanics and Engineering, 190:305-319, 2000.

[37] A. A. Johnson and T. E. Tezduyar. Mesh update strategies in parallel finite element computations of flow problems with moving boundaries and interfaces. Computer Methods in Applied Mechanics and Engineering, 119:73-94, 1994.

[38] D. Kamensky, M.-C. Hsu, D. Schillinger, J. A. Evans, A. Aggarwal, Y. Bazilevs, M. S. Sacks, and T. J. R. Hughes. An immersogeometric variational framework for fluidstructure interaction: Application to bioprosthetic heart valves. Computer Methods in Applied Mechanics and Engineering, 284:1005-1053, 2015.

[39] G. Karypis and V. Kumar. A fast and high quality multilevel scheme for partitioning irregular graphs. SIAM Journal on Scientific Computing, 20:359-392, 1999.

[40] C. E. Kees, I. Akkerman, M. W. Farthing, and Y. Bazilevs. A conservative level set method suitable for variable-order approximations and unstructured meshes. Journal of Computational Physics, 230:4536-4558, 2011.

[41] J. Kiendl, Y. Bazilevs, M.-C. Hsu, R. Wüchner, and K.-U. Bletzinger. The bending strip method for isogeometric analysis of Kirchhoff-Love shell structures comprised of multiple patches. Computer Methods in Applied Mechanics and Engineering, 199:2403-2416, 2010.

[42] J. Kiendl, K.-U. Bletzinger, J. Linhard, and R. Wüchner. Isogeometric shell analysis with Kirchhoff-Love elements. Computer Methods in Applied Mechanics and Engineering, 198:3902-3914, 2009. 
[43] J. Kiendl, M.-C. Hsu, M. C. H. Wu, and A. Reali. Isogeometric Kirchhoff-Love shell formulations for general hyperelastic materials. Computer Methods in Applied Mechanics and Engineering, 291:280-303, 2015.

[44] M. M. Koochesfahani. Vortical patterns in the wake of an oscillating airfoil. AIAA journal, 27(9):1200-1205, 1989.

[45] A. Korobenko, M.-C. Hsu, I. Akkerman, and Y. Bazilevs. Aerodynamic simulation of vertical-axis wind turbines. Journal of Applied Mechanics, 81(2):021011, 2013.

[46] S.J.C. Lai and M.F. Platzer. Jet characteristics of a plunging airfoil. AIAA journal, 37(12):1529-1537, 1999.

[47] A. Leroyer and M. Visonneau. Numerical methods for RANSE simulations of a selfpropelled fish-like body. Journal of Fluids and Structures, 20(7):975-991, 2005.

[48] C. C. Long, M. Esmaily-Moghadam, A. L. Marsden, and Y. Bazilevs. Computation of residence time in the simulation of pulsatile ventricular assist devices. Computational Mechanics, 54:911-919, 2014.

[49] C. C. Long, M.-C. Hsu, Y. Bazilevs, J. A. Feinstein, and A. L. Marsden. Fluid-structure interaction simulations of the Fontan procedure using variable wall properties. International Journal for Numerical Methods in Biomedical Engineering, 28:512-527, 2012.

[50] C. C. Long, A. L. Marsden, and Y. Bazilevs. Fluid-structure interaction simulation of pulsatile ventricular assist devices. Computational Mechanics, 52:971-981, 2013.

[51] C. C. Long, A. L. Marsden, and Y. Bazilevs. Shape optimization of pulsatile ventricular assist devices using FSI to minimize thrombotic risk. Computational Mechanics, 54:921932, 2014.

[52] A.M. Mountcastle and T.L. Daniel. Aerodynamic and functional consequences of wing compliance. Experiments in Fluids, 46(5):873-882, 2009.

[53] S.B. Raknes, X. Deng, Y. Bazilevs, D.J. Benson, K.M. Mathisen, and T. Kvamsdal. Isogeometric rotation-free bending-stabilized cables: Statics, dynamics, bending strips and coupling with shells. Computer Methods in Applied Mechanics and Engineering, 263:127$143,2013$. 
[54] D. A. Read, F. S. Hover, and M. S. Triantafyllou. Forces on oscillating foils for propulsion and maneuvering. Journal of Fluids and Structures, 17(1):163-183, 2003.

[55] Y. Saad. A flexible inner-outer preconditioned GMRES algorithm. SIAM Journal on Scientific Computing, 14(2):461-469, 1993.

[56] Y. Saad and M. Schultz. GMRES: A generalized minimal residual algorithm for solving nonsymmetric linear systems. SIAM Journal of Scientific and Statistical Computing, 7:856-869, 1986.

[57] L. Schouveiler, F. S. Hover, and M. S. Triantafyllou. Performance of flapping foil propulsion. Journal of Fluids and Structures, 20(7):949-959, 2005.

[58] W. Shyy, H. Aono, S. K. Chimakurthi, P. Trizila, C.K. Kang, C.E.S. Cesnik, and H. Liu. Recent progress in flapping wing aerodynamics and aeroelasticity. Progress in Aerospace Sciences, 46(7):284-327, 2010.

[59] K. Stein, T. Tezduyar, and R. Benney. Mesh moving techniques for fluid-structure interactions with large displacements. Journal of Applied Mechanics, 70:58-63, 2003.

[60] H. Suito, K. Takizawa, V. Q. H. Huynh, D. Sze, and T. Ueda. FSI analysis of the blood flow and geometrical characteristics in the thoracic aorta. Computational Mechanics, 54:1035$1045,2014$.

[61] K. Takizawa. Computational engineering analysis with the new-generation space-time methods. Computational Mechanics, 54:193-211, 2014.

[62] K. Takizawa, Y. Bazilevs, and T. E. Tezduyar. Space-time and ALE-VMS techniques for patient-specific cardiovascular fluid-structure interaction modeling. Archives of Computational Methods in Engineering, 19:171-225, 2012.

[63] K. Takizawa, Y. Bazilevs, T. E. Tezduyar, M.-C. Hsu, O. Øiseth, K. M. Mathisen, N. Kostov, and S. McIntyre. Engineering analysis and design with ALE-VMS and space-time methods. Archives of Computational Methods in Engineering, 21:481-508, 2014.

[64] K. Takizawa, Y. Bazilevs, T. E. Tezduyar, C. C. Long, A. L. Marsden, and K. Schjodt. ST and ALE-VMS methods for patient-specific cardiovascular fluid mechanics modeling. Mathematical Models and Methods in Applied Sciences, 24:2437-2486, 2014. 
[65] K. Takizawa, B. Henicke, A. Puntel, N. Kostov, and T. E. Tezduyar. Space-time techniques for computational aerodynamics modeling of flapping wings of an actual locust. Computational Mechanics, 50:743-760, 2012.

[66] K. Takizawa, B. Henicke, A. Puntel, N. Kostov, and T. E. Tezduyar. Computer modeling techniques for flapping-wing aerodynamics of a locust. Computers $\mathcal{E}$ Fluids, 85:125-134, 2013.

[67] K. Takizawa, N. Kostov, A. Puntel, B. Henicke, and T. E. Tezduyar. Space-time computational analysis of bio-inspired flapping-wing aerodynamics of a micro aerial vehicle. Computational Mechanics, 50:761-778, 2012.

[68] K. Takizawa, D. Montes, M. Fritze, S. McIntyre, J. Boben, and T. E. Tezduyar. Methods for FSI modeling of spacecraft parachute dynamics and cover separation. Mathematical Models and Methods in Applied Sciences, 23:307-338, 2013.

[69] K. Takizawa, D. Montes, S. McIntyre, and T. E. Tezduyar. Space-time VMS methods for modeling of incompressible flows at high Reynolds numbers. Mathematical Models and Methods in Applied Sciences, 23:223-248, 2013.

[70] K. Takizawa, K. Schjodt, A. Puntel, N. Kostov, and T. E. Tezduyar. Patient-specific computational analysis of the influence of a stent on the unsteady flow in cerebral aneurysms. Computational Mechanics, 51:1061-1073, 2013.

[71] K. Takizawa, T. Spielman, and T. E. Tezduyar. Space-time FSI modeling and dynamical analysis of spacecraft parachutes and parachute clusters. Computational Mechanics, 48:345-364, 2011.

[72] K. Takizawa, H. Takagi, T. E. Tezduyar, and R. Torii. Estimation of element-based zerostress state for arterial FSI computations. Computational Mechanics, 54:895-910, 2014.

[73] K. Takizawa and T. E. Tezduyar. Computational methods for parachute fluid-structure interactions. Archives of Computational Methods in Engineering, 19:125-169, 2012.

[74] K. Takizawa, T. E. Tezduyar, J. Boben, N. Kostov, C. Boswell, and A. Buscher. Fluidstructure interaction modeling of clusters of spacecraft parachutes with modified geometric porosity. Computational Mechanics, 52:1351-1364, 2013. 
[75] K. Takizawa, T. E. Tezduyar, C. Boswell, R. Kolesar, and K. Montel. FSI modeling of the reefed stages and disreefing of the Orion spacecraft parachutes. Computational Mechanics, 54:1203-1220, 2014.

[76] K. Takizawa, T. E. Tezduyar, C. Boswell, Y. Tsutsui, and K. Montel. Special methods for aerodynamic-moment calculations from parachute FSI modeling. Computational Mechanics, 55:1059-1069, 2015.

[77] K. Takizawa, T. E. Tezduyar, and A. Buscher. Space-time computational analysis of MAV flapping-wing aerodynamics with wing clapping. Computational Mechanics, 55:1131$1141,2015$.

[78] K. Takizawa, T. E. Tezduyar, A Buscher, and S. Asada. Space-time fluid mechanics computation of heart valve models. Computational Mechanics, 54:973-986, 2014.

[79] K. Takizawa, T. E. Tezduyar, A. Buscher, and S. Asada. Space-time interface-tracking with topology change (ST-TC). Computational Mechanics, 54:955-971, 2014.

[80] K. Takizawa, T. E. Tezduyar, and R. Kolesar. FSI modeling of the Orion spacecraft drogue parachutes. Computational Mechanics, 55:1167-1179, 2015.

[81] K. Takizawa, T. E. Tezduyar, R. Kolesar, C. Boswell, T. Kanai, and K. Montel. Multiscale methods for gore curvature calculations from FSI modeling of spacecraft parachutes. Computational Mechanics, 54:1461-1476, 2014.

[82] K. Takizawa, T. E. Tezduyar, and N. Kostov. Sequentially-coupled space-time FSI analysis of bio-inspired flapping-wing aerodynamics of an MAV. Computational Mechanics, 54:213-233, 2014.

[83] K. Takizawa, R. Torii, H. Takagi, T. E. Tezduyar, and X. Y. Xu. Coronary arterial dynamics computation with medical-image-based time-dependent anatomical models and elementbased zero-stress state estimates. Computational Mechanics, 54:1047-1053, 2014.

[84] K. Takizawa, S. Wright, C. Moorman, and T. E. Tezduyar. Fluid-structure interaction modeling of parachute clusters. International Journal for Numerical Methods in Fluids, 65:286-307, 2011.

[85] T. Tezduyar, S. Aliabadi, M. Behr, A. Johnson, and S. Mittal. Parallel finite-element computation of 3D flows. Computer, 26(10):27-36, 1993. 
[86] T. E. Tezduyar. Finite element methods for flow problems with moving boundaries and interfaces. Archives of Computational Methods in Engineering, 8:83-130, 2001.

[87] T. E. Tezduyar, M. Behr, S. Mittal, and A. A. Johnson. Computation of unsteady incompressible flows with the finite element methods - space-time formulations, iterative strategies and massively parallel implementations. In New Methods in Transient Analysis, PVP-Vol.246/AMD-Vol.143, pages 7-24, New York, 1992. ASME.

[88] T. E. Tezduyar and S. Sathe. Modeling of fluid-structure interactions with the space-time finite elements: Solution techniques. International Journal for Numerical Methods in Fluids, 54:855-900, 2007.

[89] T. E. Tezduyar, S. Sathe, R. Keedy, and K. Stein. Space-time techniques for finite element computation of flows with moving boundaries and interfaces. In S. Gallegos, I. Herrera, S. Botello, F. Zarate, and G. Ayala, editors, Proceedings of the III International Congress on Numerical Methods in Engineering and Applied Science. CD-ROM, Monterrey, Mexico, 2004.

[90] T. E. Tezduyar, S. Sathe, R. Keedy, and K. Stein. Space-time finite element techniques for computation of fluid-structure interactions. Computer Methods in Applied Mechanics and Engineering, 195:2002-2027, 2006.

[91] T. E. Tezduyar, S. Sathe, J. Pausewang, M. Schwaab, J. Christopher, and J. Crabtree. Interface projection techniques for fluid-structure interaction modeling with moving-mesh methods. Computational Mechanics, 43:39-49, 2008.

[92] T. E. Tezduyar, S. Sathe, and K. Stein. Solution techniques for the fully-discretized equations in computation of fluid-structure interactions with the space-time formulations. Computer Methods in Applied Mechanics and Engineering, 195:5743-5753, 2006. 